\title{
Article \\ Analysis of Electro-Optical Behavior in Liquid Crystal Cells with Asymmetric Anchoring Strength
}

\author{
Rumiko Yamaguchi
}

check for updates

Citation: Yamaguchi, R. Analysis of Electro-Optical Behavior in Liquid Crystal Cells with Asymmetric Anchoring Strength. Symmetry 2022, 14, 85. https://doi.org/10.3390/ sym 14010085

Academic Editors: Shoichi Ishihara and Sadahito UTO

Received: 4 December 2021

Accepted: 2 January 2022

Published: 6 January 2022

Publisher's Note: MDPI stays neutral with regard to jurisdictional claims in published maps and institutional affiliations.

Copyright: (C) 2022 by the author. Licensee MDPI, Basel, Switzerland. This article is an open access article distributed under the terms and conditions of the Creative Commons Attribution (CC BY) license (https:// creativecommons.org/licenses/by/ $4.0 /)$.
Electrical and Electronic Engineering Course, Graduate School of Engineering Science, Akita University, Akita 010-8502, Japan; yrumiko@gipc.akita-u.ac.jp; Tel.: +81-18-889-2483

\begin{abstract}
Liquid crystal director distributions have been numerically analyzed between asymmetric anchoring surfaces, that is, infinitely strong and very weak anchoring strength interfaces. In a hybrid aligned nematic (HAN) cell and a twisted nematic (TN) cell, HAN and TN orientations turn to a homogeneous orientation when the weak anchoring strength is lower than a critical one. Relationships between the anchoring strength and elastic constants of the liquid crystal were analyzed to be of a quasi-homogeneous orientation. The quasi-homogeneous orientation returned to the original HAN and TN orientations under voltage application. Low-driving electro-optical properties with no threshold voltage can be obtained in a quasi-homogeneous HAN cell. A unique voltage-transmission curve of $0-100-0 \%$ appeared in a quasi-homogeneous TN cell between the crossed polarizers.
\end{abstract}

Keywords: nematic liquid crystal; polar anchoring; azimuthal anchoring; hybrid aligned nematic; twisted nematic; homogeneous orientation; threshold voltage; transmittance

\section{Introduction}

Nematic liquid crystals (NLCs) are widely applied to information displays and optical devices of adaptive lenses, polarization grating, spatial light modulators, etc. [1]. The NLC is usually sandwiched between two glass substrates, the surfaces of which are covered with an alignment film. The alignment film surface has an easy axis and controls the NLC's orientation in the bulk of the LC cell. Conventional NLC displays are fabricated using a strong anchoring alignment surface on both sides of the substrate to maintain a specific NLC orientation. A Fréedericksz transition is one of the most famous electro-optical switching mechanisms for NLCs [2], and a typical threshold voltage is approximately 1-2 volts between strong anchoring alignment surfaces. Therefore, the driving voltage for an LC display is three times the threshold voltage or more in a typically twisted nematic (TN) display mode.

On the other hand, some types of LC displays using asymmetric anchoring strength surfaces, that is, infinitely strong and very weak anchoring alignment surfaces, have been proposed to reduce the driving voltage [3-5]. Interdigital electrodes have been fabricated on weak azimuthal anchoring substrates. A homogeneous (or TN) LC orientation in an initial state changes to a TN (or homogeneous) orientation by in-plane field, since the LC glides on the weak anchoring surface [6-8]. A polarization rotator has also been reported by using asymmetric azimuthal anchoring surfaces. LC molecules glide to the in-plane field direction on the weak anchoring surface, and LCs on the counter-strong anchoring substrate are fixed. Then, the twist angle changes, which rotates the polarization direction of the incident light [9].

Another interest for asymmetric anchoring surfaces with strong and weak anchoring strengths is the competition between the anchoring effect and elastic torque. Although the LC director prefers to align to the easy axis with the alignment surface, the actual director at the weak surface may glide from the easy axis to minimize the free energy of the LC cell, even if without an external field. The asymmetric polar anchoring strength has been 
theoretically investigated in a hybrid aligned nematic (HAN) cell. The HAN orientation changes to the homogeneous or homeotropic orientation when the thickness of the LC layer decreases to a critical thickness [10-12]. Papers have discussed the tilt angle on the weak anchoring substrate as a function of the external electric field or magnetic field. A flexo-electro-optic effect has also been investigated theoretically and experimentally in a HAN cell with asymmetric polar anchorings [13]. Moreover, Strigazzi analyzed the effect of weak azimuthal anchoring in a TN cell and the existence of the critical thickness limiting the TN orientation construction [14].

In this paper, critical anchoring is theoretically discussed instead of the critical thickness in HAN and TN cells. The LC director distributions and electro-optical properties were numerically estimated as a function of the applied voltage. A threshold-less electro-optical curve and a low-driving voltage voltage-transmission curve were obtained.

\section{Principle}

\subsection{Free Energy of LC Cells}

Figure 1 shows the definition of the liquid crystal director, $n$, with a tilt angle, $\theta$, and a twist angle, $\varphi . W_{\mathrm{p}_{-} 0}$ and $W_{\mathrm{p}_{-} \mathrm{d}}$ are the polar anchoring strengths, and $W_{\mathrm{a} \_0}$ and $W_{\mathrm{a} \_\mathrm{d}}$ are azimuthal anchoring strengths of the lower and upper substrates, respectively.

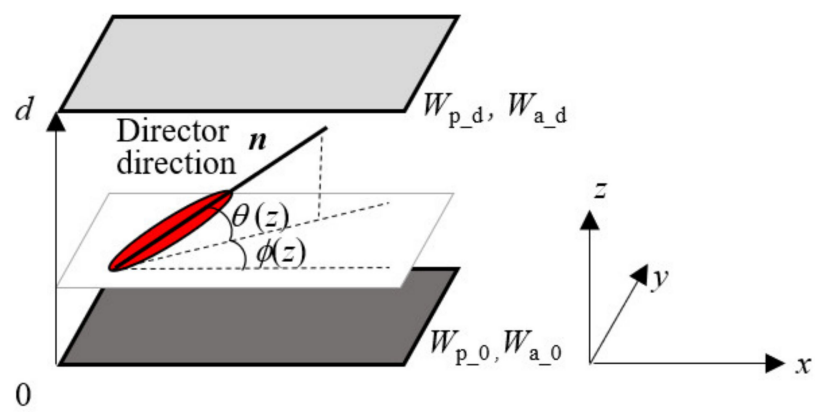

Figure 1. Definition of the liquid crystal director, $n$, and anchoring strength, $W$.

When $W_{\mathrm{p}_{0} 0}$ and $W_{\mathrm{a}_{-} 0}$ of the lower side of the substrate are strong (infinite), the total free energy per unit area, $F$, in the LC cell $[10,13,14]$ is given by:

$$
\begin{aligned}
& F=F_{\text {bulk }}+F_{\text {electric }}+F_{\text {surface }} \\
& F_{\text {bulk }}+F_{\text {electric }}=\int_{0}^{d} \frac{1}{2}\left\{\left(K_{11} \cos ^{2} \theta(z)+K_{33} \sin ^{2} \theta(z)\right)\left(\frac{d \theta(z)}{d z}\right)^{2}\right. \\
& \left.+\left(K_{22} \cos ^{2} \theta(z)+K_{33} \sin ^{2} \theta(z)\right) \cos ^{2} \theta(z)\left(\frac{d \phi(z)}{d z}\right)^{2},-\varepsilon_{0}\left(\varepsilon_{\perp}+\Delta \varepsilon \sin ^{2} \theta(z)\right)\left(\frac{d V(z)}{d z}\right)^{2}\right\} \\
& F_{\text {surface }}=\frac{1}{2} W_{\text {p_d }_{-}} \sin ^{2}\left(\theta_{\mathrm{d}}-\theta(d)\right)+\frac{1}{2} W_{\mathrm{a} \_\mathrm{d}} \sin ^{2}\left(\phi_{d}-\phi(d)\right),
\end{aligned}
$$

where $K_{11}, K_{22}$, and $K_{33}$ are the splay, twist, and bend elastic constants; $d$ is the thickness of the LC layer; $V(z)$ is the voltage potential. $\theta_{\mathrm{d}}$ and $\varphi_{\mathrm{d}}$ are easy axis angles of the upper side of the substrate. $\theta(0)$ and $\phi(0)$ at the lower side of the substrate are fixed at 0 .

In the case of the HAN cell, $\theta_{\mathrm{d}}$ is $\frac{\pi}{2}$, and $\varphi_{\mathrm{d}}$ is 0 . Without the field, the Euler-Lagrange equation leads to the splay-bend torque balance equation [13] at the homeotropic surface as follows:

$$
\left(K_{11} \cos ^{2} \theta(d)+K_{33} \sin ^{2} \theta(d)\right) \frac{\theta(d)}{d}=W_{\mathrm{p}_{-} \mathrm{d}} \sin \left(\frac{\pi}{2}-\theta(d)\right) \cos \left(\frac{\pi}{2}-\theta(d)\right) .
$$

In the TN cell, $\theta_{\mathrm{d}}$ is 0 and $\varphi_{\mathrm{d}}$ is $\frac{\pi}{2}$. Therefore, the twist torque balance equation $[9,15]$ is given by:

$$
K_{22} \frac{\varphi(d)}{d}=W_{\mathrm{a} \_\mathrm{d}} \sin \left(\frac{\pi}{2}-\varphi(d)\right) \cos \left(\frac{\pi}{2}-\varphi(d)\right) .
$$




\subsection{Critical Anchoring}

In the case of the asymmetrical anchorage effect, the upper substrate was so weak that the LC on the upper substrate could not keep the splay-bend and twist elastic torque in bulk. Consequently, the HAN and TN orientations turned to the homogeneous orientation without the orientation distortion as shown in Figure 2. The homogeneous orientation, shown in Figure 2c, may be termed as quasi-homogeneous (Q-Homo) orientation.

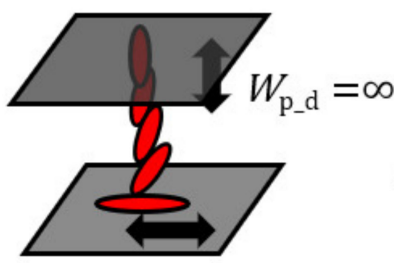

(a) HAN cell

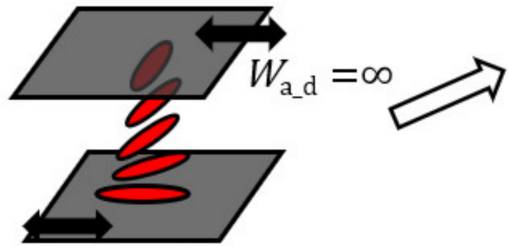

(b) TN cell
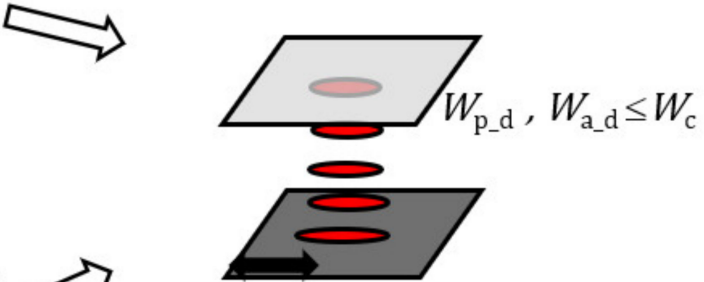

(c) Q-Homo cell

Figure 2. Schematic models of the (a) HAN, (b) TN, and (c) Q-Homo cells.

Table 1 shows the physical parameters of the LC used in the numerical calculations, which were typical values from a practical point of view. $\theta(d)$ was estimated as a function of the homeotropic polar anchoring, $W_{\mathrm{p}_{-} \mathrm{d}}$, using Equation (2) in the HAN cell as shown in Figure $3 \mathrm{a}$. The cell thickness, $d$, was $8 \mu \mathrm{m}$. $\theta(d)$ decreased with the decrease in the anchoring strength and was zero at a $W_{p_{-} d}$ of $1.5 \times 10^{-6} \mathrm{~N} / \mathrm{m}$. Such a critical value was estimated as $K_{11} / d$ and was independent of $K_{33}$ as also shown in Figure 3. The twist angle $\varphi(d)$ also decreased with decreasing $W_{a_{-} d}$ in the TN cell as shown in Figure $3 \mathrm{~b}$. The critical anchoring was estimated as $K_{22} / d\left(=1.0 \times 10^{-6} \mathrm{~N} / \mathrm{m}\right)$ using Equation (3). The change in the twist angle was experimentally confirmed by controlling the anchoring of a poly(vinyl cinnamate) film [15].

Table 1. Physical parameters of the LC.

\begin{tabular}{ccccccc}
\hline$K_{\mathbf{1 1}}$ & $\boldsymbol{K}_{\mathbf{2 2}}$ & $\boldsymbol{K}_{\mathbf{3 3}}(\mathbf{p N})$ & $\varepsilon_{/ /}$ & $\varepsilon_{\perp}$ & $n_{\mathbf{o}}$ & $n_{\mathbf{e}}$ \\
\hline 12 & 8 & 18 & 15 & 5 & 1.5 & 1.7 \\
\hline
\end{tabular}
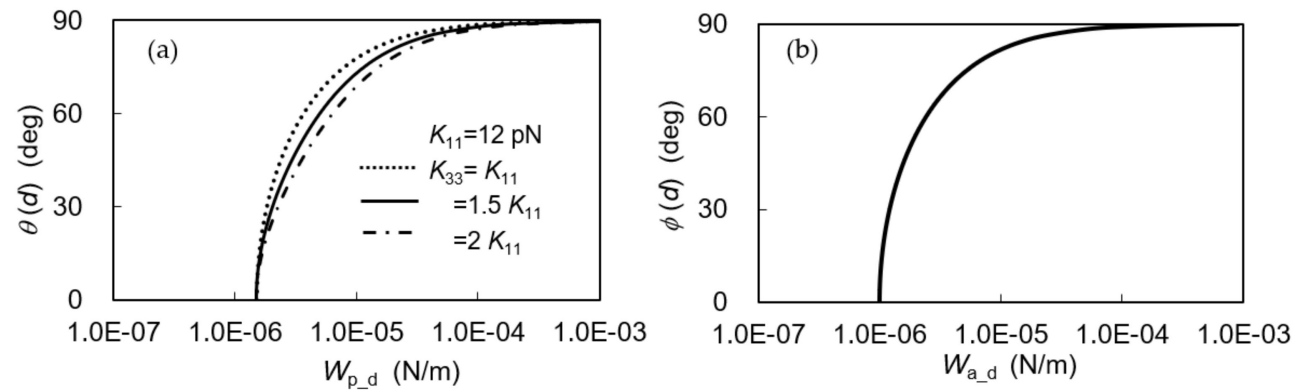

Figure 3. (a) $W_{p_{-} d}$ vs. tilt angle $\theta(d)$ in the HAN cell and (b) $W_{a_{-} d}$ vs. twist angle $\varphi(d)$ in the TN cell.F. 


\section{Electro-Optical Property in the Q-Homo Cell}

\subsection{Q-Homo-HAN Cell}

Figure 4 shows $\theta(d)$ as a function of the applied voltage in the HAN cell with the parameter of $W_{p_{-} \mathrm{d}}$. The LC's physical properties, as shown in Table 1, were used in this section. $\theta(d)$ increased with the applied voltage and reached $90^{\circ}$ at an applied voltage higher than $3 \mathrm{~V}$. In the Q-Homo state of the HAN (Q-Homo-HAN) cell with an anchoring of $1.5 \times 10^{-6} \mathrm{~N} / \mathrm{m}\left(=W_{\mathrm{c}}\right), \theta(d)$ increased from $0^{\circ}$ without the threshold voltage. The threshold voltage, $V_{\text {th }}$, appeared when $W_{\text {p_d }}$ was less than $W_{\mathrm{c}} . V_{\text {th }}$ was $0.48 \mathrm{~V}$ in the cell when $W_{\text {p_d }}$ was $0.1 W_{\mathrm{c}}=1.5 \times 10^{-6} \mathrm{~N} / \mathrm{m}$.

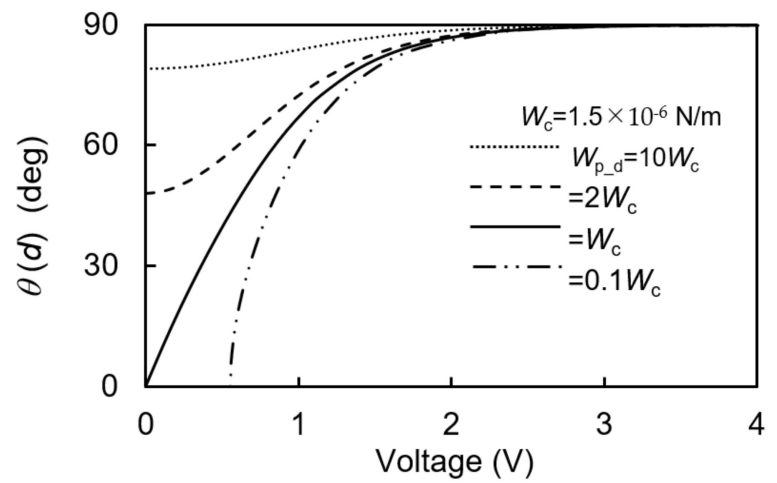

Figure 4. $\theta(d)$ as a function of the applied voltage.

Figure 5a shows the LC director distributions of $\theta$ in the Q-Homo-HAN cell with the parameter of the applied voltage. $W_{\mathrm{p}_{-} \mathrm{d}}$ was $1.5 \times 10^{-6} \mathrm{~N} / \mathrm{m}\left(=W_{\mathrm{c}}\right)$. The LC tilt angle at the center of the cell was $30^{\circ}$ by applying a voltage of only $0.5 \mathrm{~V}$. Moreover, the $\theta$ distribution at $4 \mathrm{~V}$ was almost the same as that in the conventional HAN cell with infinite anchoring on both sides of the substrate. From these curves, an effective extraordinary refractive index $<n_{\mathrm{e}}>$ was estimated as follows:

$$
<n_{e}>=\frac{1}{d} \int_{0}^{d} \frac{n_{e} n_{o}}{\sqrt{\cos ^{2} \theta(z) n_{o}^{2}+\sin ^{2} \theta(z) n_{e}^{2}}} d z,
$$

where $n_{\mathrm{o}}$ and $n_{\mathrm{e}}$ are ordinary and extraordinary indices, respectively. Figure $5 \mathrm{~b}$ shows $<n_{\mathrm{e}}>$ vs. applied voltage in Q-Homo-HAN cells with a $W_{\mathrm{p}_{-} \mathrm{d}}$ of $W_{\mathrm{c}}$ and $0.1 W_{\mathrm{c}}$. Conventional Homo and HAN cells are also shown in Figure 5a. The Homo cell had a threshold voltage of approximately $1.16 \mathrm{~V}$ when using the LC parameters shown in Table 1 . In the Q-Homo-HAN cell with $W_{\mathrm{c}},<n_{\mathrm{e}}>$ decreased without the threshold voltage and was approximately 1.53 at $2 \mathrm{~V}$, which was almost the same value as the HAN cell. Therefore, a large index change of approximately 0.17 was obtained in the Q-Homo-HAN cell compared to 0.06 in the HAN cell and 0.10 in the Homo cell at $2 \mathrm{~V}$.

(a)

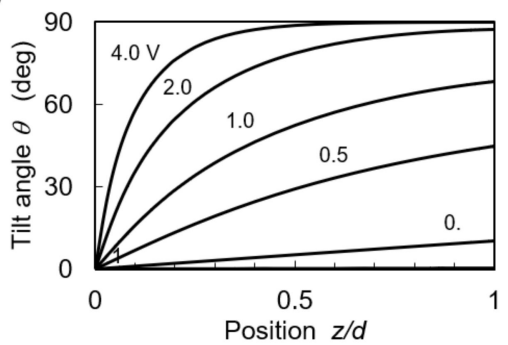

(b)

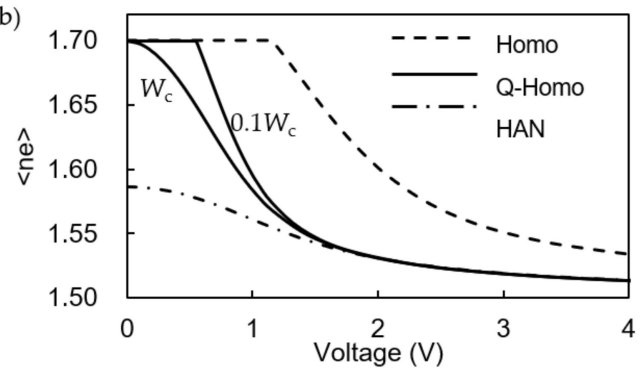

Figure 5. (a) LC director distributions of $\theta$ in the Q-Homo-HAN with a $W_{\mathrm{p}_{-} \mathrm{d}}$ of $1.5 \times 10^{-6} \mathrm{~N} / \mathrm{m}$ $\left(=W_{\mathrm{c}}\right)$. (b) Effective extraordinary refractive index vs. applied voltage in Q-Homo, HAN, Homo, and HAN cells. 
A guest-host (GH) mode of the electro-optical property in the Q-Homo-HAN cell was also compared with the HAN and Homo cells. Typical absorption coefficients of 0.03 and $0.3 \mu \mathrm{m}^{-1}$ for short and long axes of a dichroic dye were used, respectively [16]. The incident light was polarized parallel to the LC director of the planar aligned surface. In this case, the maximum (homeotropic orientation) and the minimum (homogeneous orientation) transmittances were estimated at 78.7 and 9.1\%, respectively. Figure 6 shows transmittance vs. applied voltage curves in three cells. In the HAN cell, the transmittance in the voltage on state was higher than that in the Homo cell. However, the transmittance in the off state was also high, and the contrast ratio was very low. The transmittance in the on state of the Homo cell was lower, since the LCs did not reorient on both the strong anchoring planar surfaces. The driving voltage, $V_{90}$, at which the transmittance increased to $T_{90}(=\sim 72 \%)$ was approximately $13 \mathrm{~V}$. On the other hand, $V_{90}$ could decrease to $7 \mathrm{~V}$ in the Q-Homo-HAN cell.

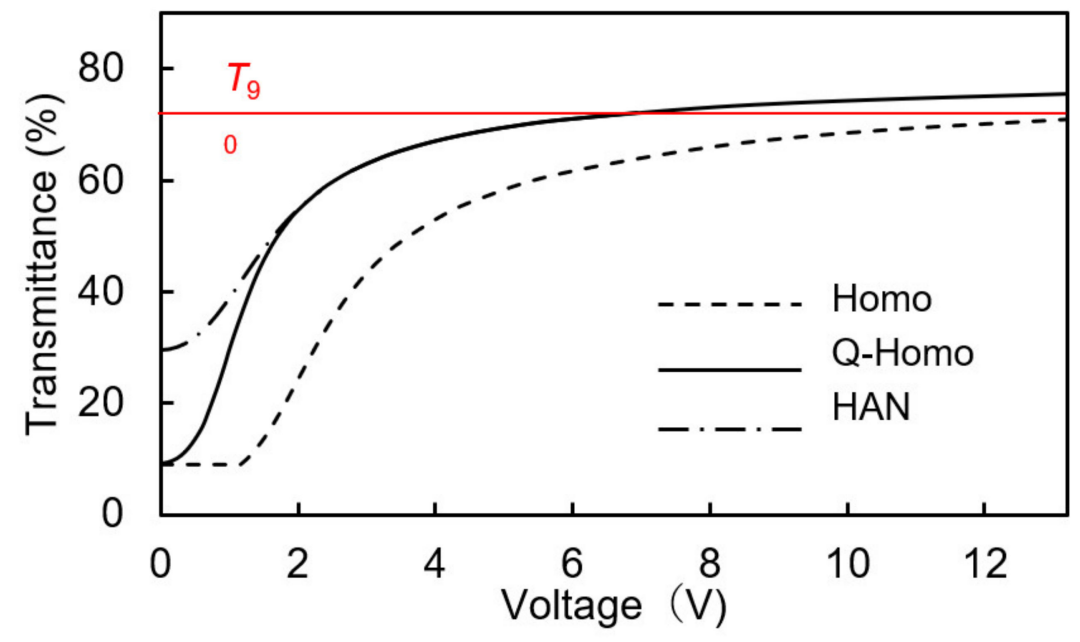

Figure 6. Transmittance as a function of the applied voltage in the GH mode.

\subsection{Q-Homo-TN Cell}

Figure 7 shows $\varphi(d)$ as a function of the applied voltage in the TN cell with the parameter of $W_{\text {a_d. }}$. Elastic constants and dielectric constants shown in Table 1 were used in this section. The polar anchoring of both sides of the substrate was infinite. $\varphi(d)$ on the weak azimuthal anchoring surface increased with the applied voltage, since the twist torque in bulk decreased with the reorientation of the LC perpendicular to the substrate. Therefore, curves had a threshold voltage which corresponded to the voltage when the tilt angle started to increase. $V_{\text {th }}$ was $1.16 \mathrm{~V}$ in the TN cell as well as in the homogeneous cell when using the cell parameter shown in Table 1 . When $W_{\mathrm{a} \_\mathrm{d}}$ was less than $W_{\mathrm{c}}$, for example, $0.5 W_{\mathrm{c}}, V_{\text {th }}$ increased to approximately $2.4 \mathrm{~V} . \varphi(d)$ turned to the original easy axis $\varphi_{\mathrm{d}}$ of $\frac{\pi}{2}$ by applying a voltage of more than $5 \mathrm{~V}$. Figure 8 shows the schematic models of LC director distribution when the voltage was applied. Its starts from the homogeneous orientation to the TN orientation, and I call this type of LC cell a Q-Homo-TN cell.

Tilt and twist angle distributions were numerically estimated with the applied voltages and are shown in Figure 9. The polar anchoring strength was infinite and the pretilt angles $\theta(0)$ and $\theta(d)$ were $0^{\circ}$. The distributions of $\theta$ with the respective applied voltages were almost the same as those in a conventional $\mathrm{TN}$ cell using both strong anchoring surfaces. On the other hand, the $\varphi$ distribution changed from the homogeneous state to the TN state. Both the $\theta$ and $\varphi$ distributions were exactly the same as those in the conventional TN at $5.0 \mathrm{~V}$. 


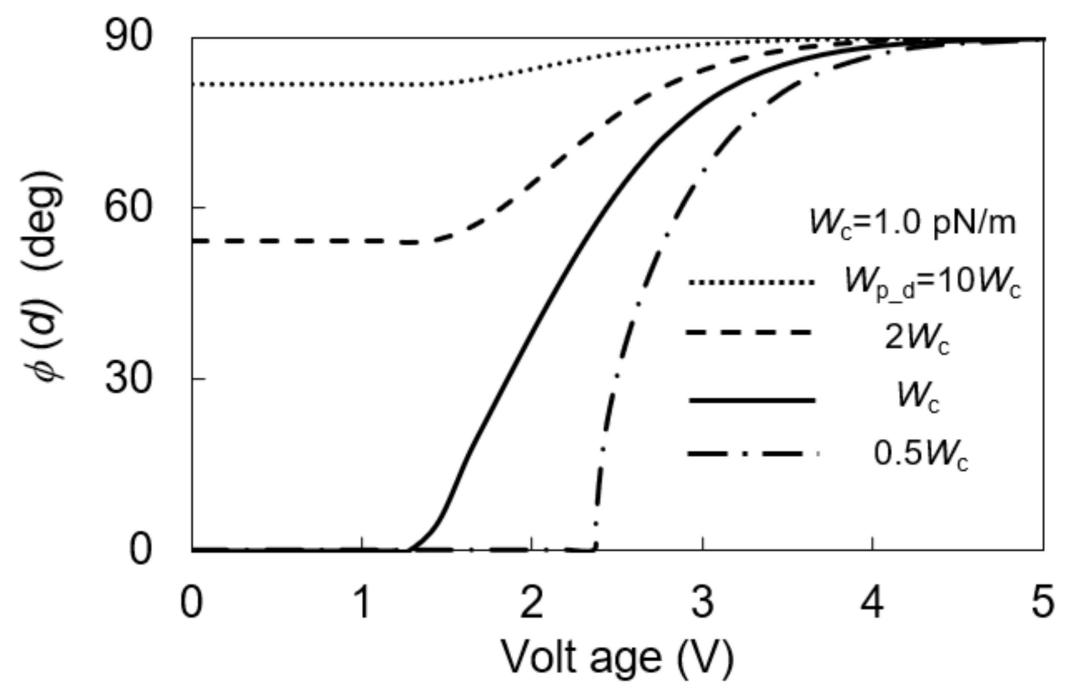

Figure 7. $\varphi(d)$ as a function of the applied voltage.
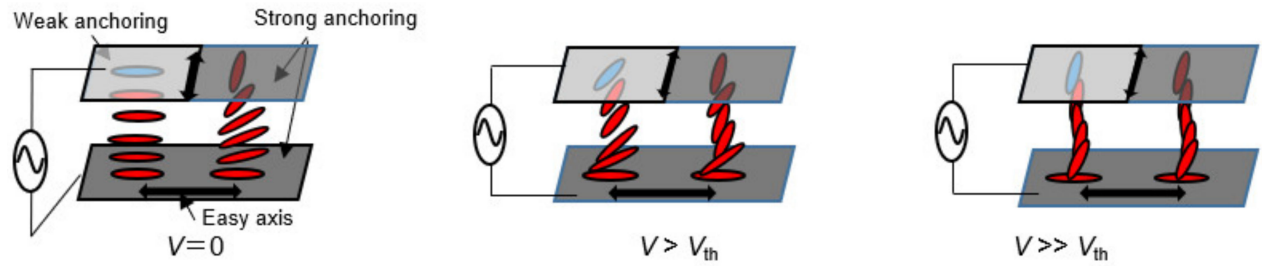

Figure 8. Schematic model of LC director distribution under the respective applied voltages in Q-Homo-TN cell (left side) and the conventional TN (right side) cell.

(a)

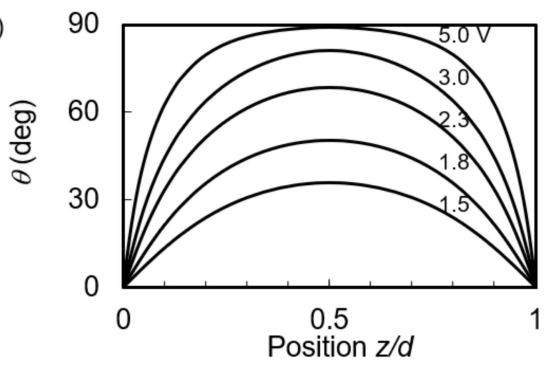

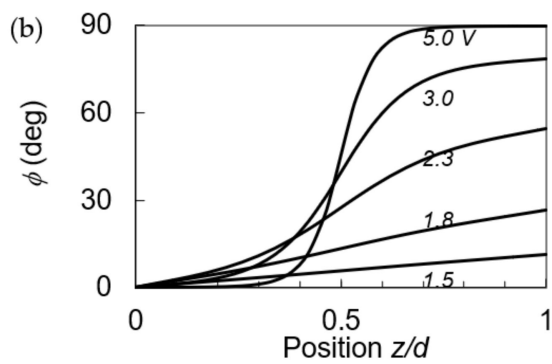

Figure 9. Director distributions of (a) $\theta$ and (b) $\varphi$.

Subsequently, the voltage-transmittance curves of the cell between the crossed polarizers were estimated using Jones matrix calculus $[17,18]$ as shown in Figure 10a. The wavelength was $550 \mathrm{~nm}$, which is very close to the third minimum condition; therefore, the transmittance in the voltage off state was $100 \%$ in the $90^{\circ} \mathrm{TN}$ cell. When the voltage was applied, the LC director reoriented along the electric field as shown in Figure 9a, and the twist angle distribution also changed. Thus, the polarization guiding effect was destroyed, and the transmittance reduced to $0 \%$. On the other hand, in the Q-Homo-TN cell, it increased from 0 to $98.4 \%$ at $2.35 \mathrm{~V}$, which was approximately two times that of the threshold voltage. Then, it decreased to $0 \%$ again because of the same circumstances of the TN cell. IF $\Delta n$ is $0.18,100 \%$ transmittance can be obtained at $2.31 \mathrm{~V}$. The polarization states of the transmission light with the respective applied voltages are also shown in Figure 10b. When the lineally polarized light parallel to the $x$-axis was incident on the cell, the major axis of the elliptical polarized transmission light rotated to the $y$-axis when the voltage increased to $2.3 \mathrm{~V}$ and then returned to the $x$-axis again when the voltage increased to $4.0 \mathrm{~V}$. 
(a)

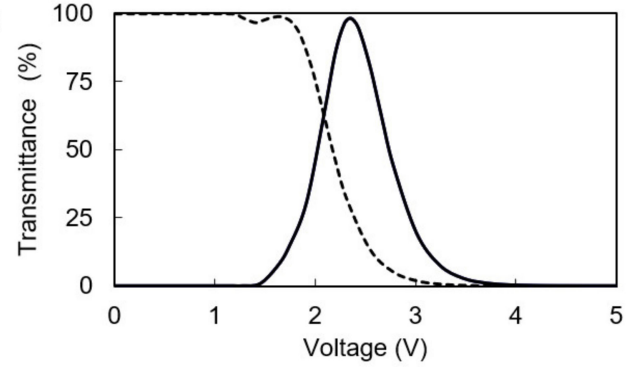

(b)

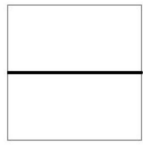

$0 \mathrm{~V}$

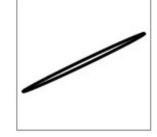

$18 \mathrm{~V}$

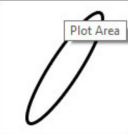

$2.1 \mathrm{~V}$

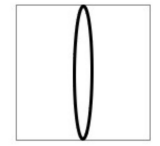

$2.3 \mathrm{~V}$

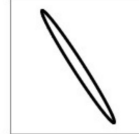

$2.6 \mathrm{~V}$

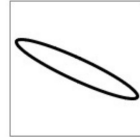

$3.0 \mathrm{~V}$

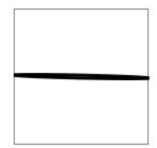

$4.0 \mathrm{~V}$

Figure 10. (a) Voltage-transmittance curves in Q-Homo-TN and TN cells. (b) Polarization states of the transmission light in the Q-Homo-TN cell with the respective applied voltages.

\section{Conclusions}

Electro-optical properties were numerically analyzed in liquid crystal cells with asymmetric anchoring strengths. The hybrid orientation turned to the homogeneous orientation when the polar anchoring of the homeotropic alignment surface reduced to the critical anchoring of $K_{11} / d$. The quasi-homogeneous HAN cell had no threshold voltage. Larger optical variations could be obtained by using a lower applied voltage compared to the conventional homogeneous cell. The twisted orientation turned to the homogeneous orientation when the azimuthal anchoring of the one side of the planar alignment surface reduced to the critical anchoring of $K_{22} / d$. The transmittance of the quasi-homogeneous TN cell between the crossed polarizers increased from $0 \%$ to approximately $100 \%$ at a voltage of approximately $2 V_{\text {th }}$. Liquid crystal display modes with low-driving voltages can be clarified by using asymmetric anchoring strength.

Funding: This research received no external funding.

Institutional Review Board Statement: Not applicable.

Informed Consent Statement: Not applicable.

Data Availability Statement: Not applicable.

Conflicts of Interest: The author declares no conflict of interest.

\section{References}

1. Jaroszewicz, L.; Bennis, N. Liquid Crystal Optical Device; MDPI: Basel, Switzerland, 2020. [CrossRef]

2. Frederick, V.K.; Zolina, V. Forces causing the orientation of an anisotropic liquid. Trans. Faraday Soc. 1933, 29, 919-930. [CrossRef]

3. Yoneya, M.; Iwasaki, K.; Tomioka, Y.; Kondo, K. Cell gap margin enlargement of in-plane switching mode liquid crystal displays using weak-anchoring effects. Appl. Phys. Lett. 1999, 74, 803-805. [CrossRef]

4. Bryan-Brown, G.P.; Wood, E.L.; Sage, I.C. Weak surface anchoring of liquid crystals. Nature 1999, 399, 338-340. [CrossRef]

5. Andrienko, D.; Barbet, F.; Bormann, D.; Kurioz, Y.; Kwon, S.-B.; Reznikov, Y.; Warenghem, M. Electrically controlled director slippage over a photosensitive aligning surface; in-plane sliding mode. Liq. Crysts. 2000, 27, 365-370. [CrossRef]

6. Sato, O.; Iwata, N.; Kawamura, J.; Maeda, T.; Tsujii, Y.; Watanabe, J.; Tokita, M. An in-plane switching liquid crystal cell with weakly anchored liquid crystals on the electrode substrate. J. Mater. Chem. C 2017, 5, 4384-4387. [CrossRef]

7. Choi, Y.; Oh, S.-W.; Choi, T.-H.; Sohn, H.-J.; Do, S.-M.; Yoon, T.-H. Liquid crystal cell asymmetrically anchored for high transmittance and triggered with a vertical field for fast switching. Opt. Express 2020, 28, 20553-20562. [CrossRef] [PubMed]

8. Sato, O.; Okuno, H.; Adachi, I.; Goto, K.; Noda, T.; Tsutsui, K. A high transmittance and fast response in-plane switching liquid crystal display with the zero-azimuth anchoring layers on the electrodes. J. Phys. D Appl. Phys. 2020, 53, 15LT02. [CrossRef]

9. Yamaguchi, R.; Yamanaka, T.; Sato, S. Liquid-Crystal Optical Rotator Using Weak Azimuthal Anchoring Surface. Jpn. J. Appl. Phys. 2001, 40, 6522-6525. [CrossRef]

10. Barbero, G.; Barberi, R. Critical thickness of a hybrid aligned nematic liquid crystal cell. J. Phys. 1983, 44, 609-616. [CrossRef]

11. Barbero, G.; Bartolino, R. Remarks on critical thickness of a hybrid aligned nematic cell with large elastic anisotropy. Mol. Cryst. Liq. Cryst. 1983, 99, 89-97. [CrossRef] 
12. Strigazzi, A. Second order elasticity and critical thickness of hybrid aligned nematics strongly anchored on the planar side. Mol. Cryst. Liq. Cryst. 1990, 179, 425-433. [CrossRef]

13. Madhusudana, N.V.; Durand, G. Linear flexo-electro-optic effect in a hybrid aligned nematic liquid crystal cell. J. Phys. Lett. 1985, 46, 195-200. [CrossRef]

14. Strigazzi, A. On the critical thickness of a twisted nematic cell. J. Phys. 1985, 46, 1507-1512. [CrossRef]

15. Yamaguchi, R.; Sato, S. Continuous grey scale image printing on the liquid crystal cell. Appl. Phys. Lett. 2005, 86, 031913. [CrossRef]

16. Uchida, T.; Wada, M. Guest-Host Type Liquid Crystal Displays. Mol. Cryst. Liq. Cryst. 1981, 63, 19-44. [CrossRef]

17. Allia, P.; Oldano, C.; Trossi, L. Polarization transfer matrix for the transmission of light through liquid-crystal slabs. J. Opt. Soc. Am. B 1988, 5, 2452-2461. [CrossRef]

18. Lien, A. Extended Jones matrix representation for the twisted nematic liquid-crystal display at oblique incidence. Appl. Phys. Lett. 1990, 57, 2767. [CrossRef] 